\title{
Kollokationen im Lernerwörterbuch - Anspruch und Wirklichkeit
}

\author{
Jupp Möhring (Leipzig)
}

\begin{abstract}
This article outlines an approach which explores how frequent lexical collocations can be identified for a representative extract of the German lexicon. It takes into account corpuslinguistic and phraseological criteria. The lexical foundation for this study is a corpus-driven and thematically-classified frequency dictionary of German. Additionally, the extensive DWDS corpus and a manual linguistic selection were used to identify typical collocations. Finally, after identifying 56 high-frequency collocations, four of the foremost learner dictionaries in the field of German as a foreign language were analyzed quantitatively for the presence of these collocations.
\end{abstract}

\section{$1 \quad$ Einführung}

Die Relevanz lexikalischer Kollokationen für Fremdsprachenlerner, in diesem Artikel bezogen auf jene des Deutschen als Fremdsprache, wurde bereits mehrfach deutlich herausgestellt (cf. Hausmann 1984: 400ff; Herbst 1996: 389ff, Reder 2006: 59ff; Steyer 2008: 186; Wallner 2010: 197f.) und wird deshalb in diesem Beitrag ebenso wenig nochmals diskutiert wie der Terminus Kollokation selbst (cf. dazu u. a. Bahns 1996: 6ff; Bubenhofer 2009: 111ff; Hausmann 2004; Konecny 2010: 77ff; Steyer 2008: 187ff). Auch die sich aus der Lernerrelevanz folgerichtig ergebende lernerlexikographische Forderung, Kollokationen "nicht länger als zufälliges Accessoire eines Wörterbucheintrages [zu] betrachten" (Viehweger 1989: 892), "da sie einen Datentyp darstellen, der einem vorrangigen Benutzerbedürfnis entspricht" (Viehweger 1989: 892 nach Steyer 2000: 105), ist schon gestellt (cf. Bahns 1996: 37f.) und die Beschreibung der Darstellung von Kollokationen in verschiedenen Wörterbüchern konsequenterweise ein wichtiger Aspekt der Wörterbuchkritik (cf. u. a. Engelberg/Lemnitzer 2001: 189ff; Köster/Neubauer 2002; Lehr 1998; Markus/Korhonen 2005; Reder 2006: 167 ff; Steyer 2008: 191ff). Jedoch scheinen nicht nur die Wörterbuchautoren Probleme bei der Kollokationsauswahl und -verzeichnung zu haben, denn auch "[d]ie Untersuchung der Kollokationen in den Lernerwörterbüchern geschieht im allgemeinen mit relativ geringer Systematik" (Jehle 1990: 117). Was aber genau unter einer ausführlichen und systematischen Analyse $\mathrm{zu}$ verstehen ist, wird in der einschlägigen Literatur sehr unterschiedlich definiert (cf. Bahns 1996: 68). Dies führt dazu, dass die Auswahl des zu überprüfenden Lexikonausschnitts oft auf vagen Kriterien basiert (cf. Jehle 1990: 264).

Dieser Beitrag möchte einen Vorschlag unterbreiten, wie semantische, phraseologische und korpuslinguistische Kriterien kombiniert für eine systematische Wörterbuchanalyse genutzt werden können. So werden besonders gebräuchliche und somit lernerrelevante lexikalische Kollokationen für einen Ausschnitt des Lexikons identifiziert. Im Anschluss daran werden 
vier $^{1}$ einsprachige Lernerwörterbücher (LWB) quantitativ ${ }^{2}$ auf die Eintragung dieser Kollokationen hin untersucht.

\section{$2 \quad$ Kollokationen}

Als Kollokationen werden in diesem Beitrag binäre ${ }^{3}$ Wortverbindungen gewertet, welche aus einer nominalen Basis und einem verbalen Kollokator bestehen (cf. Hausmann 1984: 401; Reder 2001: 2). Andere Strukturtypen (cf. Bahns 1993: 33) bleiben unberücksichtigt. Kollokationen sind nicht satzwertig, semantisch kompositionell, strukturell variant und weisen häufig eine eingeschränkte Kombinationsfähigkeit ${ }^{4}$ auf (Neubert 1966: 109; Reder 2004: 532f.). Diese eingeschränkte Kombinierbarkeit ist von besonderer Relevanz für den Fremdsprachenlerner, da dadurch sprachvergleichend häufig unvorhersehbare Kontraste in der Kombinationsfähigkeit (Scherfer 2001: 9) und somit Interferenzfehler auftreten.

\section{$3 \quad$ Auswahl der Kollokationen}

\subsection{Die Ausgangswörter}

Um die Aussagefähigkeit der Wörterbuchanalyse zu gewährleisten, wurde auf thematische Vielfalt und hohe Gebräuchlichkeit der Kollokationen geachtet.

Thematische Vielfalt wurde gewährleistet, indem der onomasiologisch geordnete Grundwortschatz "Grund- und Aufbauwortschatz Deutsch als Fremdsprache nach Themen" (Tschirner 2008b) genutzt wurde. Dieser Wortschatz umfasst die 4000 häufigsten Wörter des Deutschen ${ }^{5}$, eingeordnet in 16 Sachgruppen. Diese sind an Profile Deutsch (Glabionat 2010) und somit am Gemeinsamen europäischen Referenzrahmen für Sprachen orientiert. Als Ausgangswörter für die Kollokationsidentifikation wurden aus dem "Grund- und Aufbauwortschatz Deutsch als Fremdsprache nach Themen" (Tschirner 2008b) die häufigsten Nomen der 16 Sachgruppen sowie die zehn häufigsten Verben aus der Sonderkategorie die 50 häufigsten Verben extrahiert. Diese Auswahl hat gegenüber Auswahlmethoden, welche z. B. eine breite alphabetische Streuung (cf. Bahns 1996: 63f.; Jehle 1990: 259ff; Markus/Korhonen 2005) oder die erfolgte Aufnahme einer Mehrwortverbindung in einem anderen Wörterbuch als Kriterium definieren, wesentliche Vorteile. So ist die alphabetische Verteilung des Wortschatzes sehr ungleichmäßig (cf. Haß-Zumkehr 2001: 383f.) und der Eintrag in einem Wörterbuch keine Garantie für die tatsächliche Gebräuchlichkeit. Häufigkeitsaspekte spielen hierbei eine ungleich relevantere Rolle (cf. Tschirner 2005: 134ff). Die Kollokationsidentifikation auf Grundlage der 16 Nomen wird im Folgenden baseninduzierte Analyse genannt.

Neben der baseninduzierten Analyse wurde eine kollokatoreninduzierte Analyse durchgeführt, wobei die zehn häufigsten Verben des Deutschen die Datengrundlage bildeten6 .

${ }^{1}$ Der damit einhergehende quantitative Vergleich der Kollokationsaufnahme der relevantesten einsprachigen LWB ist ein Desiderat der Wörterbuchforschung, welche i.d.R. lediglich ein WB gründlich analysiert, gelegentlich noch Vergleiche zu einem anderen (L)WB zieht, jedoch bislang nicht auf einer empirisch verlässlichen Datenbasis auf alle vier LWB reflektiert.

2 Zu weiteren Analyseebenen cf. Steyer 2008: 193.

3 Die Beschränkung auf binäre Einheiten wird vor allem durch die weitere Arbeit mit dem DWDS Korpus notwendig, da die dort angebotene Kollokationsanalyse nur für binäre Einheiten verfügbar ist.

${ }^{4}$ Zu möglichen Ursachen dieser cf.: Karacic 2004: 293; Kromann 1989: 267; Nesselhauf 2005: 30f.; Reder 2004: 534 und Scherfer 2001: 18.

5 Die empirische Grundlage dafür bildet das 4,2 Millionen Token große, ausgewogene und repräsentative Herder/BYU-Korpus der deutschen Gegenwartssprache (Jones/Tschirner 2006; Tschirner 2008a).

6 Von der Analyse unbeachtet blieben darunter Hilfsverben, Modalverben und Funktionsverben, da eine Analyse von verbnominalen Verbindungen in einem Korpus bei Verben, welche im Deutschen zur Bildung von Zeitformen genutzt werden, kaum zielführende Aussagekraft besitzt, sollte das Korpus nicht in die 
Tabelle 1 zeigt die als Analysebasis genutzten Nomen und Verben mit der Angabe des Ranks ${ }^{7}$ im thematisch geordneten Frequenzwörterbuch.

\begin{tabular}{|c|c|c|}
\hline Name der Sachgruppe & Rank im WB & Ausgangswort \\
\hline \multicolumn{3}{|r|}{ Nomen } \\
\hline 1. Personalien, Informationen zur Person & 103 & Frau \\
\hline 2. Wohnen & 159 & Haus \\
\hline 3. Umwelt & 186 & Stadt \\
\hline 4. Reisen und Verkehr & 104 & Mensch \\
\hline 5. Verpflegung & 970 & Essen $^{8}$ \\
\hline 6. Einkaufen & 251 & Geld \\
\hline 7. Öffentliche und private Dienstleistungen & 350 & System \\
\hline 8. Körper und Gesundheit & 179 & Hand \\
\hline 9. Wahrnehmung und Bewegung & 323 & Sinn \\
\hline 10. Ausbildung & 89 & Beispiel \\
\hline 11. Arbeitswelt & 167 & Arbeit \\
\hline 12. Sprache & 231 & Grund $^{9}$ \\
\hline 13. Freizeit und Unterhaltung & 204 & Ende \\
\hline 14. Persönliche Beziehungen und Kontakte & 305 & Entwicklung \\
\hline 15. Politik und Gesellschaft & 160 & Fall \\
\hline 16. Allgemeine Begriffe & 51 & Jahr \\
\hline 17. Strukturwörter & & Kein Nomen \\
\hline
\end{tabular}

(Fortsetzung auf der nächsten Seite)

Funktionen des Verbs als Vollverb oder Hilfsverb unterscheiden können. Dies ist bei der Analyse mit Hilfe des DWDS Kernkorpus nicht möglich. Die Funktionsverben wurden von der Analyse ausgeschlossen, da viele ihrer signifikanten nominalen Partner mit ihnen tatsächlich Funktionsverbgefüge bilden. Die Analyse zielt jedoch darauf ab, eine möglichst große Anzahl hochfrequenter lexikalischer Kollokationen zu finden, um deren Darstellung in den großen einsprachigen LWB zu überprüfen. Als Funktionsverben gelten hier nach Heine (2004: 74): bringen, finden, gehen, halten, kommen, nehmen, setzen, stehen, stellen, treten und ziehen. Aus technischen Gründen musste außerdem das Verb machen von der Analyse ausgeschlossen werden, da hierfür keine Daten in der Kollokationsdatenbank des DWDS vorliegen.

${ }^{7}$ Der Rank gibt die Platzierung in der Häufigkeitsliste an, wobei Rank 1 das häufigste Wort definiert, Rank 2 das zweithäufigste usw. Der Rank ergibt sich aus der absoluten Häufigkeit eines Lexems inkl. aller Nennformen innerhalb des Herder/BYU-Korpus. Ist der Wert der Häufigkeit zwischen mehreren Lexemen identisch, so wird alphabetisch geordnet.

8 Das Nomen Essen wird in "Deutsch als Fremdsprache nach Themen" nicht separat aufgeführt, sondern lediglich das Verb essen (Rank 657). Die Zuordnung erfolgte manuell.

${ }^{9}$ Für eine ausführliche Beschreibung von Kollokationen mit der Basis Grund cf. Steyer 2008: 198ff. 


\begin{tabular}{|c|c|c|}
\hline 18. 50 häufigste Verben & & Verb \\
\hline & 46 & sagen \\
\hline & 57 & geben \\
\hline & 81 & sehen \\
\hline & 82 & lassen \\
\hline & 112 & bleiben \\
\hline & 118 & liegen \\
\hline & 123 & heißen \\
\hline & 124 & denken \\
\hline & 140 & tun \\
\hline & 143 & glauben \\
\hline
\end{tabular}

Tabelle 1: Die als Analysebasis genutzten Nomen und Verben

Insgesamt wurden somit 26 Ausgangswörter für die anschließende Kollokationsidentifikation genutzt, welche einen thematisch ausgewogenen Ausschnitt des Wortschatzes darstellen. Zudem gehören sie durchweg zu den 1000 häufigsten Wörtern des Deutschen und sind somit essenziell für jeden Fremdsprachenlerner (cf. Tschirner 2008a: 195).

\subsection{Die Kollokationsidentifikation}

Für die Identifikation der statistisch signifikantesten Kollokationspartner wurde das DWDS Kernkorpus ${ }^{10}$ genutzt, da es mit 100 Millionen laufenden Textwörtern der Mindestgröße zur Analyse von Mehrworteinheiten (Tschirner 2005: 137) entspricht und für die Analyse von Wörterbüchern geeignet ist (Geyken 2004: 74, 79). Es ist repräsentativ aufgebaut, stellt gut geeignete Recherchewerkzeuge bereit und ermöglicht, bis auf wenige, urheberrechtliche Einschränkungen, Zugang zu bibliografisch markierten Belegen ${ }^{11}$.

Mit Hilfe der Kollokationsabfrage des DWDS wurden im Rahmen der baseninduzierten Analyse die gemäß dem Assoziationsmaß Log-Likelihood ${ }^{12} 20$ signifikantesten verbalen Kollokationspartner zu jedem Nomen identifiziert. Für die kollokatoreninduzierte Analyse wurden auf die gleiche Weise die 20 signifikantesten nominalen Kollokationspartner gefunden. Die Kollokationssuche bei DWDS erfolgte in einer Kollokationsspanne von jeweils fünf Wörtern links und rechts des untersuchten Ausgangswortes. Für die 26 Ausgangswörter lagen somit insgesamt 520 statistisch signifikante Kollokationen (cf. Tabellen im Anhang) ${ }^{13}$ vor. Diese Wortkombinationen beinhalteten jedoch nicht ausschließlich lexikalische Kollokationen, sondern auch freie Wortverbindungen (worunter auch alle semantisch und/oder grammatisch nicht verbundenen Kombinationen gezählt werden), Funktionsverbgefüge und Idiome, weshalb anhand der eingangs erwähnten Kriterien manuell

10 Verfügbar unter http://www.dwds.de, Stand 30. Mai 2010.

11 Das DWDS ist eins von inzwischen mehreren großen verfügbaren Korpora der deutschen Sprache, die sich zur Kollokationssuche eignen. An dieser Stelle sei ergänzend auf das Deutsche Referenzkorpus DeReKo des IDS Mannheim und das Wortschatzportal der Universität Leipzig verwiesen (cf. Steyer 2008: 189ff).

12 Das Log-Likelihood (LL) gilt als das zuverlässigste Maß zur Identifikation von lexikalischen Kollokationen (McEnery/Tono/Xiao 2006: 55f., 217).

13 Diese 520 Kookkurrenzen (zur terminologischen Abgrenzung cf. Steyer 2008: 187ff) bilden die quantitative Basis der Untersuchung. Sie sind dem Artikel als Anhang beigefügt. Da es sich beim DWDS Kernkorpus um ein statisches Korpus handelt, sind sie auch jederzeit online nachprüfbar. 
überprüft und selektiert werden musste. Die Überprüfung der Kombinationen hinsichtlich der lexikalischen Kriterien von Kollokationen ergab eine relativ geringe Menge typischer Kollokationen, was Reder (2006: 62) zufolge auf Grund der weitgehenden semantischen Verträglichkeit und Kombinationsfähigkeit im Grundwortschatz zu erwarten war. Zu einigen Ausgangswörtern konnte innerhalb der Untersuchungsmenge gar keine lexikalische Kollokation gefunden werden. So sind die häufigsten verbalen Partner ${ }^{14}$ von Frau: sagen, sehen, kommen, lieben, sitzen, gehen, fragen, müssen, stehen, geben, sollen, lassen, wissen, sprechen, rufen, erzählen, nehmen, denken, kennen, dürfen, bitten. Die einzige offenkundige Besonderheit bei diesen Wortpaaren tritt in der Struktur (zur) Frau nehmen auf, wobei dieses Wortpaar eindeutig zu den Idiomen gezählt werden muss, da die Gesamtbedeutung heiraten sich nicht aus den einzelnen Komponenten erschließt.

Insgesamt konnten 59 häufige ${ }^{15}$ lexikalische Kollokationen identifiziert werden. In zwei Fällen fand sich die gleiche hochfrequente und als Kollokation eingeordnete Wortkombination sowohl bei der baseninduzierten als auch bei der kollokatoreninduzierten Untersuchung. Es handelt sich dabei um: Beispiel geben und Grund geben. Für die Analyse der Wörterbücher war eine doppelte Verzeichnung nicht notwendig, weshalb die beiden Kollokationen in diesem Teil der Auswertung nur jeweils einmal geführt werden. Ihre Zugehörigkeit zum hochfrequenten Wortschatzbereich ist durch die Dopplung unterstrichen. Eine weitere Auffälligkeit der baseninduzierten Analyse war das Auftreten von laden und einladen in Kombination mit Essen. Die Sichtung der Belege ergab, dass laden in der Struktur einladen genutzt wird. Die Korpusanalyse hat die Trennung des Verbs in ein- und laden nicht erfassen können. Für die Analyse der Wörterbücher wird lediglich die Struktur Essen einladen untersucht. Entsprechend wird Essen laden als Kollokation nicht berücksichtigt. Analog wird mit den Kollokatoren treten und eintreten bei der Basis Haus verfahren. Es bleiben 56 hochfrequente lexikalische Kollokationen, welche im Folgenden, alphabetisch aufsteigend nach ihrer Basis sortiert, aufgelistet sind ${ }^{16}$ :

Anlass geben, Arbeit gehen, Arbeit geben, Aufschluss geben, Auskunft geben, Beispiel geben, Beispiel folgen, Beispiel anfïhren, Beispiel liefern, Ende setzen, Ende kommen, Ende finden, Entwicklung sehen, Erinnerung bleiben, Essen einladen, Essen gehen, Essen servieren, Fall eintreten, Gedächtnis bleiben, Gefallen tun, Geld verdienen, Geld kosten, Geld ausgeben, Geld sparen, Geld stecken, Geld lassen, Gelegenheit geben, Genüge tun, Grund geben, Grund sehen, Hand halten, Hand drücken, Hand heben, Hand geben, Hand schütteln, Hände falten, Haus schicken, Haus treten/eintreten, Haus gehen, Interesse liegen, Leben bleiben, Leben lassen, Mühe geben, Pflicht tun, Ruhe lassen, Schnee liegen, Schritt tun, Schwerpunkt liegen, Sinn geben, Sinn kommen, Stadt ziehen, Unrecht tun, Verhältnis liegen, Wahrheit sagen, Wasser lassen, Zeit lassen.

Da die Abfrage der Kollokationsdatenbank des DWDS sich auf binäre Einheiten beschränkt, kann an dieser Stelle nicht differenziert werden, ob die Kollokationen präferiert mit einem oder mehreren weiteren Lexem/en, bspw. Präpositionen, auftreten.

\footnotetext{
14 Vor der Reduktion um Modal- und Funktionsverben.

${ }^{15}$ Häufig sei verstanden als gemäß LL-Wert und somit neben anderen Parametern auch gemäß der Häufigkeit signifikant.

16 Die unscharfe Grenzziehung in der Kategorisierung von Mehrwortverbindungen und in der Auslegung einzelner Kriterien lässt an dieser Stelle zweifellos eine Diskussion über die Zuordnung der einen oder anderen lexikalischen Kollokation zu. So könnte man bspw. der Wortverbindung Genüge tun einen gewissen Grad an Unikalität/Idiomatizität unterstellen oder den Grad der Kompostionalität der Verbindungen Wasser lassen oder Mühe geben diskutieren. Dennoch sind all diese Wortverbindungen statistisch sehr gebräuchlich und auch die identifizierten Kollokationspartner gehören bis auf die Ausnahmen Aufschluss und falten zu den häufigsten 4000 Wörtern des Deutschen.
} 


\section{$4 \quad$ Analyse der Wörterbücher}

Nach der Identifikation der 56 hochfrequenten Kollokationen zu den 26 Ausgangswörtern wurde das Vorkommen dieser Kollokationen in den vier großen einsprachigen LWB des Deutschen als Fremdsprache analysiert. Es handelt sich dabei um das de Gruyter Wörterbuch Deutsch als Fremdsprache (GWB), das Langenscheidt Großwörterbuch Deutsch als Fremdsprache (LDaF), das Pons Großwörterbuch Deutsch als Fremdsprache (PDaF) und das Wahrig Großwörterbuch Deutsch als Fremdsprache (WAR). Die Wörterbücher wurden jeweils auf einen Eintrag der Kollokation unter der nominalen Basis und unter dem verbalen Kollokator der Kollokation überprüft. Somit ergeben sich 112 mögliche Einträge der 56 untersuchten Kollokationen.

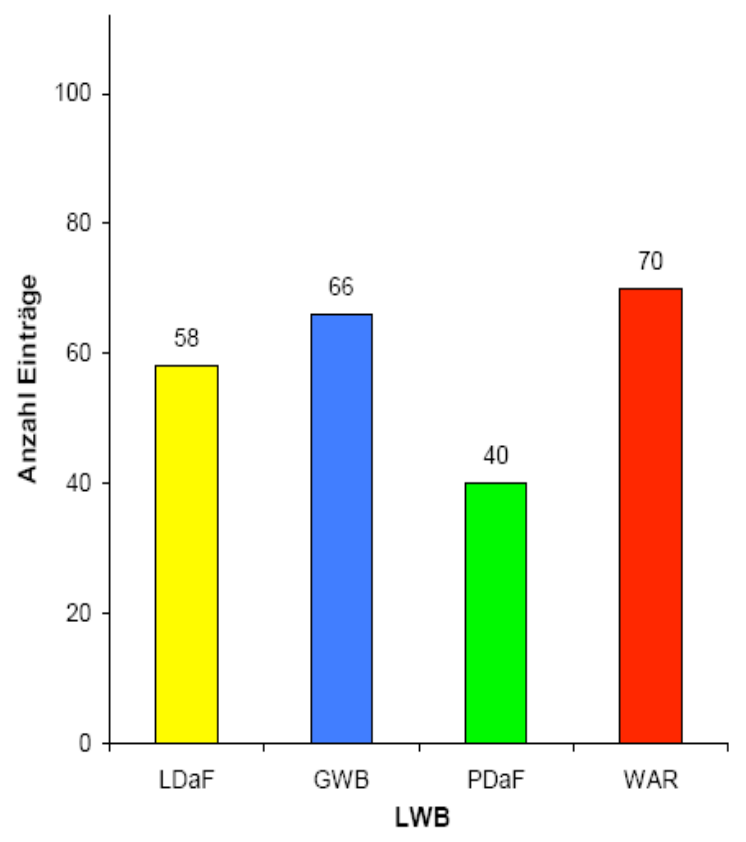

Abb. 1: Anzahl der Kollokationseinträge von 112 möglichen Eintragungen im LWB

In diesem Beitrag wird dabei nicht differenziert, in welchem Teil der Wörterbuchartikel ${ }^{17}$ die Kollokationen auftreten. Als Eintrag gewertet wurden zudem alle möglichen Formen, in denen die Kollokationen in den LWB verzeichnet sind, da die Korpusanalyse keine präzisere Aussage zu über die Zweiworteinheit hinausgehenden Kombinationskonventionen der Kollokationen (Numerus, Präpositionen, Konjunktionen, Pronomen) (siehe oben) treffen konnte $^{18}$. Das quantitative Darstellungsoptimum von 112 Einträgen erreicht keins der untersuchten LWB. Den höchsten Wert bietet das WAR mit insgesamt 70 Einträgen, etwa ein Drittel der möglichen Eintragungen liefert das PDaF (Abb.1). Die Differenz von 30 Einträgen zwischen dem LWB mit den meisten und den wenigsten verzeichneten Kollokationen verdeutlicht, wie uneinheitlich die Aufnahme von Kollokationen in die LWB schon bei einem kleinen Ausschnitt des Grundwortschatzes erfolgt ${ }^{19}$. Im Folgenden wird

\footnotetext{
17 Natürlich ist für eine Aussage über die Qualität der Kollokationsdarstellung der LWB auch die Mikrostruktur der Wörterbuchartikel zu berücksichtigen (cf. Steyer 2008: 193f.), sprich ob die Kollokationen im Beispielteil oder Definitionsteil des Artikels erscheint, ob sie als Idiom oder Kollokation gekennzeichnet sind etc. Dieser Artikel beschränkt sich jedoch auf quantitative Aspekte und somit das WAS, für Vorschläge zum WIE cf. u. a. Bahns 1996: 39ff, Steinbügl 2005: 46ff und Steyer 2008: 200f.

18 Zum Desiderat der an dieser Stelle vernachlässigten Berücksichtigung von Flexionsmerkmalen und Erweiterungskonventionen von Kollokationen cf. Steyer 2008: 197ff.

19 Wobei die Anzahl der eingetragenen Stichworte zwischen WAR (70 000) und PDaF (77 000) vergleichbar ist.
} 
genauer analysiert, ob die Kollokationen unter dem nominalen oder/ und dem verbalen Stichwort verzeichnet sind.

Für einen Eintrag der Kollokation beim verbalen Kollokator spricht, dass dieser ohne die Angabe der kollokierenden Basen gar nicht in all seinen Lesarten erfasst werden könnte (Bahns 1996: 39). Zudem kann ein solcher Eintrag helfen, "synonyme Lexeme in ihrer Bedeutung und Verwendungsweise zu differenzieren" (Bahns 1996: 40). Häufig erschweren gerade die Kollokatoren das Verständnis von Kollokationen (Jehle 1990: 262), so dass aus rezeptiver Sicht ein Eintrag unter dem Kollokator wünschenswert wäre (ebd.). Wörterbücher, in denen unter Kollokatoren "Informationen über mögliche Basen und Basisklassen" (Hausmann 2007: 219) eingetragen sind, sind kognitionsorientiert (ebd.).

Für einen Eintrag von Kollokatoren unter der jeweiligen Basis spricht, dass der L2Wörterbuchbenutzer bei der Textproduktion weit häufiger nach einem passenden Kollokator zu einer ihm bereits bekannten Basis sucht, als umgekehrt (cf. Jehle 1990: 116). Demnach werden WB dieser Struktur auch als produktionsorientiert bezeichnet. Gesuchte Kollokatoren findet der Nutzer beim Nachschlagen unter dem ihm bekannten Autosemantikon (Hausmann 2007: 218). Zudem ist natürlich nicht auszuschließen, dass auch die Basis, obgleich autosemantisch und somit auch ohne Kollokator definierbar, gerade im Falle einer Kollokation Verständnisprobleme bereitet.

Es ist also eine Vielzahl produktiver und rezeptiver Nutzungssituationen denkbar, in denen der Eintrag einer Kollokation unter ihren beiden Einzellexemen nützlich wäre (cf. Bahns 1996, 110; Reder 2006: 183).

Folgt man der Annahme, dass lexikalische Kollokationen rezeptiv unproblematisch sind und der Lerner für produktive Bedürfnisse nach passenden Kollokatoren im LWB suchen wird, sollten die 56 Kollokationen zumindest unter den nominalen Basen zu finden sein (cf. Jehle 1990: 263). Tendenziell scheinen die LWB diesem Prinzip durchaus zu folgen (cf. Abb. 2), denn von allen Kollokationen, die nur unter der Basis oder dem Kollokator in den LWB eingetragen sind, finden sich durchweg mehr ausschließlich unter der Basis. In allen LWB finden sich jedoch auch ausschließlich unter dem Kollokator eingetragene Kollokationen und sowohl unter Basis als auch unter dem Kollokator eingetragene Mehrwortverbindungen. Diese Uneinheitlichkeit ist für den Benutzer eines LWB sehr nachteilig, denn er kann sich nicht darauf verlassen, die Kollokation entweder unter dem Nomen, unter dem Verb oder gar unter beiden finden zu können. Die Werte zeigen, dass ein eindeutiges Konzept hinsichtlich des Eintragungsorts nicht vorhanden ist.

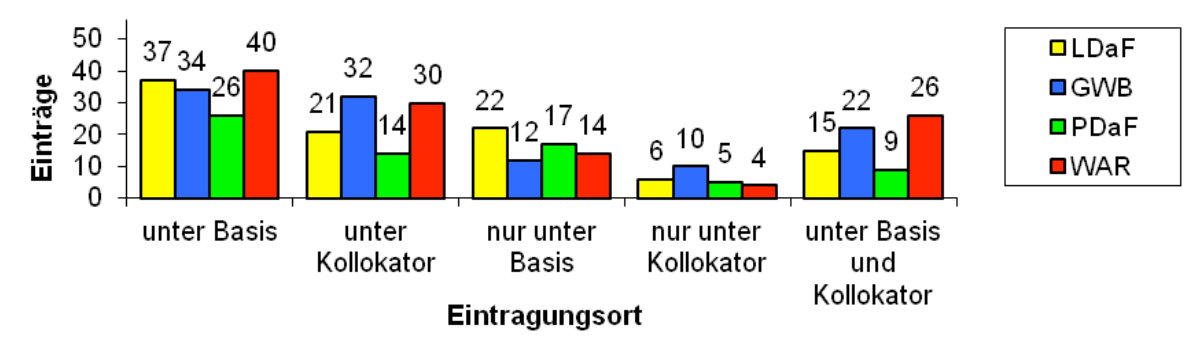

Abb. 2: Darstellung der Kollokationen nach Eintragungsort 


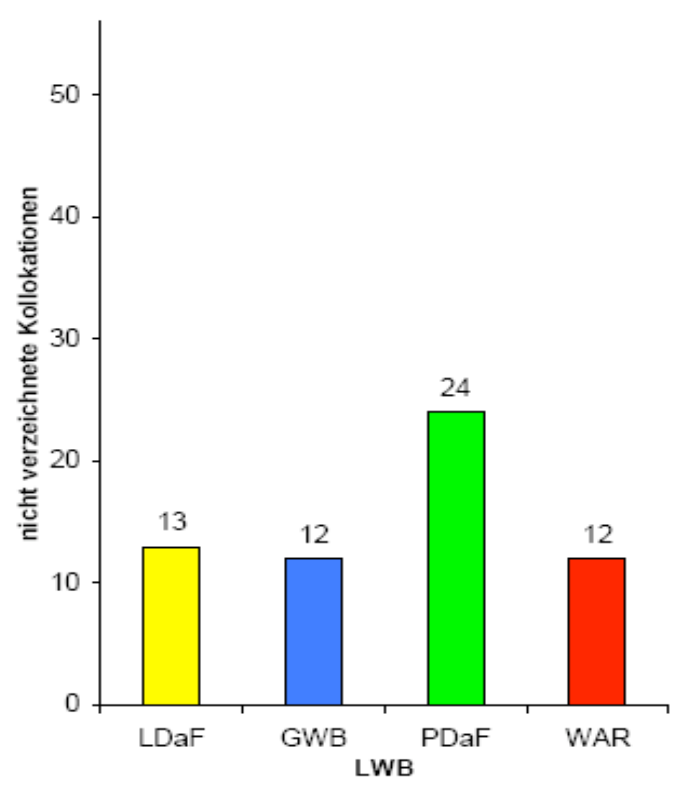

Abb. 3: Anzahl der nicht in den LWB eingetragenen Kollokationen

In allen LWB fehlen einige der 56 Kollokation (cf. Abb.3), wobei lediglich vier der hochfrequenten Kollokationen (Entwicklung sehen, Geld stecken, Grund sehen, Verhältnis liegen) in keinem der LWB verzeichnet sind, alle anderen sind in mindestens einem der LWB eingetragen. Dieser Wert zeigt zum einen, dass die durch die aufwendige Kollokationsidentifikation gefundenen Mehrwortverbindungen von den Wörterbuchautoren ebenfalls als relevant erachtet werden, wenn auch auf nicht nachvollziehbar unterschiedliche Art und Weise. Zum anderen wird klar, dass die insgesamt überhaupt nicht im LWB verzeichneten Kollokationen (cf. Abb. 3), wovon es bis zu 24 von 56 gibt, ein tatsächlich großes Desiderat bei der Entwicklung aktueller LWB darstellen. Fünf Kollokationen sind in jedem der untersuchten LWB sowohl unter der Basis als auch unter dem Kollokator eingetragen (Geld sparen, Geld verdienen, Hand halten, Hand schütteln, Hause gehen). Diese Schnittmenge sollte wesentlich größer ausfallen, wenn alle LWB auf der Basis großer, ausgewogener und repräsentativer Korpora erstellt worden wären.

\section{$5 \quad$ Fazit}

Es wurde für einen repräsentativen Lexikonausschnitt gezeigt, wie hochfrequente und somit zum Grundwortschatz gehörige Kollokationen im Korpus datengeleitet identifiziert werden können. Diese Auswahlmethode ermöglicht es, systematisch für den Fremdsprachenlerner tatsächlich relevante Mehrwortverbindungen zu benennen. Im Anschluss wurden die vier großen LWB des Bereichs Deutsch als Fremdsprache auf die Erfassung dieser Kollokationen hin untersucht und verglichen.

In der Wörterbuchanalyse beschränkt sich der geleistete Beitrag v.a. auf quantitative Aspekte der lexikographischen Erfassung. Dies lässt eine abschließende Bewertung, welches der LWB eine besonders/weniger gelungene Kollokationsdarstellung aufweist, nicht zu. Die durchweg unvollständige und uneinheitliche Verzeichnung der betrachteten Kollokationen impliziert jedoch für die weitere lexikographische Arbeit Forderungen, wie sie bereits Heine (2005: 353) für den verwandten Bereich der Funktionsverbgefüge gestellt hat:

Für weitere Wörterbücher bzw. Neuauflagen ist es wünschenswert, die mittlerweile vielfältigen Möglichkeiten, die die Arbeit mit elektronischen Korpora bietet, zu nutzen. Nur so können die bislang auf intuitiver und damit subjektiver Muttersprachen-Kompetenz von Autoren und Informanten beruhenden Angaben im Wörterbuch [...] durch objektivere, dem tatsächlichen Sprachgebrauch entsprechende, ergänzt oder ersetzt werden. 
Auch Steyer (2008: 192) fordert, dass "[j]edem lexikografischen Arbeitsprozess [...] heute Korpusanalysen und im Idealfall auch Kookkurrenzanalysen vorgelagert sein" sollten. Insbesondere für den in diesem Beitrag untersuchten Bereich des Grundwortschatzes, welcher für den Lerner auf einem Sprachniveau erwerbsrelevant ist, auf dem er das LWB seines Vertrauens häufig nutzt, zeigen die Resultate, dass lexikographisch nachgebessert werden muss.

Dabei gilt es, neben der Auswahl und Aufnahme der relevanten Kollokationen auch eine konsequente, transparente und für den Lerner nützliche Lösung bezüglich des Eintragungsortes zu finden. Dem unter 4. geäußerten Vorschlag, Kollokationen sowohl unter der Basis als auch unter dem Kollokator zu verzeichnen, setzt der beschränkte Umfang von Printwörterbüchern eine natürliche Grenze. Eine Kollokation benötigt im Wörterbuch doppelt so viel Raum, wenn sie unter beiden Komponenten eingetragen ist. Gebräuchliche Komprimierungsverfahren wie bspw. die lexikographische Beschränkung auf das beteiligte Substantiv (cf. Steyer 2000, 106f.) sind jedoch kaum geeignet, bestehende Lernerbedürfnisse zu befriedigen. Konsequente Verweise zwischen den einzelnen Einträgen böten an dieser Stelle einen, wenn auch nicht unproblematischen, Kompromiss zwischen Platzökonomie und umfassender Registrierung an (ebd.), doch zumindest für den Bereich des Grundwortschatzes sollte eine umfassende Verzeichnung der Kollokationen angestrebt werden. Die Rechtfertigung unvollständiger Verzeichnung wichtiger sprachlicher Phänomene durch räumliche Begrenzung ist in Zeiten aufkommender elektronischer Wörterbücher jedoch ohnehin ein scheidendes Argument (cf. Haß-Zumkehr 2001: 378f.).

Die angesprochenen lexikographischen Fragestellungen Welche Kollokationen sollten im LWB eingetragen sein? und Wo sollten diese Kollokationen verzeichnet sein? müssen dem Wie? (cf. Kap.4) voran gestellt sein und jeder dieser Aspekte bedarf zweifellos weiterer wissenschaftlicher Betrachtung. Es ist wünschenswert, dass all diese Punkte in der weiteren lernerlexikographischen Arbeit stärkere Beachtung finden um den rezeptiven und produktiven Bedürfnissen der Lerner gerecht zu werden.

\section{Literatur}

\section{Wissenschaftliche Literatur}

Bahns, Jens (1993): "Kollokation kontra Kontext. Wider ein zu weites Verständnis des Kollokationsbegriffs". Praxis des neusprachlichen Unterrichts 40: 30-37.

Bahns, Jens (1996): Kollokationen als lexikographisches Problem. Eine Analyse allgemeiner und spezieller Lernerwörterbücher des Englischen. Tübingen: Niemeyer. (=Lexicographica Series maior 74).

Bubenhofer, Noah (2009): Sprachgebrauchsmuster. Korpuslinguistik als Methode der Diskurs- und Kulturanalyse. Berlin: de Gruyter. (=Sprache und Wissen).

Engelberg, Stefan/Lemnitzer, Lothar (2001): Lexikographie und Wörterbuchbenutzung. Tübingen: Stauffenburg-Verlag. (=Stauffenburg Einführungen 14).

Geyken, Alexander (2004): "Korpora als Korrekiv für einsprachige Wörterbücher". Zeitschrift für Literaturwissenschaft und Linguistik 136: 72-100.

Glaboniat, Manuela (2010): Profile deutsch. Gemeinsamer europäischer Referenzrahmen; Lernzielbestimmungen, Kannbeschreibungen, kommunikative Mittel; Niveau A1 - A2, B1 B2, $C 1$ - C2. Berlin: Langenscheidt.

Haß-Zumkehr, Ulrike (2001): Deutsche Wörterbücher - Brennpunkt von Sprach- und Kulturgeschichte. Berlin/New York: de Gruyter.

Hausmann, Franz Josef (1984): "Wortschatzlernen ist Kollokationslernen. Zum Lehren und Lernen französischer Wortverbindungen". Praxis des neusprachlichen Unterrichts 4/31: 395-406. 
Hausmann, Franz Josef (2004): "Was sind eigentlich Kollokationen?". In: Steyer, Kathrin (ed.): Wortverbindungen - mehr oder weniger fest. Berlin, de Gruyter: 309-334.

Hausmann, Franz Josef (2007): "Die Kollokationen im Rahmen der Phraseologie. Systematische und historische Darstellung". Zeitschrift für Anglistik und Amerikanistik 3/55: 217-234.

Heine, Antje (2004): "Funktionsverbgefüge in System, Text und korpusbasierter (Lerner-)Lexikografie." Dissertationsschrift zur Erlangung des Doktorgrades Doctor philosophiae (Dr. phil.). Leipzig: Universität Leipzig.

Heine, Antje (2005): "Funktionsverbgefüge im Lernerwörterbuch. Ein Vergleich von "Langenscheidt Großwörterbuch Deutsch als Fremdsprache" (2003) und "De Gruyter Wörterbuch Deutsch als Fremdsprache." In: Barz, Irmhild/Bergenholtz, Henning/Korhonen, Jarmo (eds.): Schreiben, Verstehen, Übersetzen, Lernen. Zu ein- und zweisprachigen Wörterbüchern mit Deutsch. Frankfurt am Main, P. Lang: 345-356.

Herbst, Thomas (1996): "What are Collocations: Sandy Beaches or False Teeth?". English Studies 77: 379-393.

Jehle, Günter (1990): Das englische und französische Lernerwörterbuch in der Rezension. Theorie und Praxis der Wörterbuchkritik. Tübingen: Niemeyer. (=LexicographicaSeries maior 30).

Karacic, Geriena (2004): "Verträglichkeit und Unverträglichkeit der Kollokate". In: Bracic, Stojan (ed.): Linguistische Studien im Europäischen Jahr der Sprachen. Akten des 36. Linguistischen Kolloquiums in Ljubljana $2001=$ Linguistic studies in the European year of languages. Frankfurt am Main, Lang: 291-300.

Konecny, Christine (2010): "Lexikalische Kollokationen und der Beitrag der InternetSuchmaschine Google zu ihrer Erschließung und Beschreibung". In: Ptashnyk, Stefaniya/ Hallsteinsdóttir, Erla/Bubenhofer, Noah (eds.): Korpora, Web und Datenbanken. Computergestützte Methoden in der modernen Phraseologie und Lexikographie = Corpora, Web and Databases: computer-based methods in modern phraseology and lexicography. Baltmannsweiler, Schneider Verlag Hohengehren: 77-94.

Köster, Lutz/Neubauer, Fritz (2002): "Kollokationen und Kompetenzbeispiele im De Gruyter Wörterbuch Deutsch als Fremdsprache". In: Wiegand, Herbert Ernst (ed.): Perspektiven der pädagogischen Lexikographie des Deutschen II. Untersuchungen anhand des "de Gruyter Wörterbuchs Deutsch als Fremdsprache". Tübingen, Niemeyer: 283-310.

Kromann, Hans-Peder (1989): "Zur Funktionalen Beschreibung von Kollokationen in Übersetzungswörterbüchern". In: Gréciano, Gertrud (ed.): Europhras 88. Phraséologie constrastive ; actes du Colloque international, Klingenthal-Strasbourg, 12 - 16 mai 1988. Strasbourg, Univ. des Sciences Humaines Dép. d'Etudes Allemandes: 265-271.

Lehr, Andrea (1998): "Kollokationen in Langenscheidts Großwörterbuch Deutsch als Fremdsprache". In: Wiegand, Herbert Ernst (ed.): Perspektiven der pädagogischen Lexikographie des Deutschen. Untersuchungen anhand von "Langenscheidts Grosswörterbuch Deutsch als Fremdsprache": Tübingen, Niemeyer: 256-281.

Markus, Tuulikki/Korhonen, Jarmo (2005): "Kollokationen in der deutschen Lernerlexikographie und in deutsch-finnischen Wörterbüchern". In: Barz, Irmhild/Bergenholtz, Henning/Korhonen, Jarmo (eds.): Schreiben, Verstehen, Übersetzen, Lernen. Zu ein- und zweisprachigen Wörterbüchern mit Deutsch. Frankfurt am Main, P. Lang: 327-344.

McEnery, Tony/Tono, Yukio/Xiao, Richard (2006): Corpus-based language studies. An advanced resource book. London: Routledge. (=Routledge applied linguistics).

Nesselhauf, Nadja (2005): Collocations in a learner corpus. Amsterdam etc.: Benjamins. (=Studies in corpus linguistics 14).

Neubert, Albrecht (1966): "Analogien zwischen Phonologie und Semantik". In: Hintze, Fritz/Meier, Georg Friedrich/Seidel, Eugen (eds.): Zeichen und System der Sprache. 
Veröffentlichung des 2. Internationalen Symposions "Zeichen und System der Sprache " vom 8. 9. bis 15.9.1964 in Magdeburg. Berlin, Akademie Verlag: 106-116.

Reder, Anna (2001): "Ein heißer Tipp - oder Kollokationslernen durch eine Ganzschrift". In: Häcki Buhofer, Annelies/Durco, Peter (eds.): Wortschatz: Aneignung und Unterricht. IDT Publikation 2001. http://www.sprachwissenschaft.ch/IDT2001/, Stand 30.05.2010

Reder, Anna (2004): "Eine Frage aufwerfen oder: Kollokation als lexikographisches Problem in Wörterbüchern". In: Bracic, Stojan (ed.): Linguistische Studien im Europäischen Jahr der Sprachen. Akten des 36. Linguistischen Kolloquiums in Ljubljana 2001 = Linguistic studies in the European year of languages. Frankfurt am Main, Lang: 531-542.

Reder, Anna (2006): Kollokationen in der Wortschatzarbeit. Wien: Praesens-Verlag.

Scherfer, Peter (2001): "Zu einigen wesentlichen Merkmalen lexikalischer Kollokationen". In: Lorenz-Bourjot, Martine/Lüger, Heinz-Helmut (eds.): Phraseologie und Phraseodidaktik. Wien, Ed. Praesens: 4-19.

Steinbügl, Birgit (2005): Deutsch-englische Kollokationen. Erfassung in zweisprachigen Wörterbüchern und Grenzen der korpusbasierten Analyse. Tübingen: Niemeyer. (=LexicographicaSeries maior 126).

Steyer, Kathrin (2000): "Usuelle Wortverbindungen des Deutschen. Linguistisches Konzept und lexikografische Möglichkeiten.". Deutsche Sprache 2/28: 101-125.

Steyer, Kathrin (2008): "Kollokationen in deutschen Wörterbüchern und in der deutschen Wörterbuchforschung". In: Heid, Ulrich et al. (eds.): Lexicographica 24. International Annual for Lexicography - Revue Internationale de Lexicographie - Internationales Jahrbuch für Lexikographie. Berlin, New York: de Gruyter: 185-208.

Tschirner, Erwin (2005): "Korpora, Häufigkeitslisten, Wortschatzerwerb". In: Heine, Antje/Hennig, Mathilde/Tschirner, Erwin (eds.): Deutsch als Fremdsprache - Konturen und Perspektiven eines Faches. Festschrift für Barbara Wotjak zum 65. Geburtstag. München, Iudicium: 133-152.

Tschirner, Erwin (2008a): "Das professionelle Wortschatzminimum im Deutschen als Fremdsprache". Deutsch als Fremdsprache 4/45: 195-208.

Viehweger, Dieter (1989): "Probleme der Beschreibung semantischer Vereinbarkeitsrelationen im allgemeinen einsprachigen Wörterbuch". In: Hausmann, Franz Josef et al. (eds.): Wörterbücher. Ein internationales Handbuch zur Lexikographie. Berlin; New York: de Gruyter: 888-893.

Wallner, Franziska (2010): "Kollokationen in Wissenschaftssprachen: Zur lernerlexikographischen Relevanz der Textarten- und Diskursspezifik von Kollokationen". In: Ptashnyk, Stefaniya/ Hallsteinsdóttir, Erla /Bubenhofer, Noah (eds.): Korpora, Web und Datenbanken. Computergestützte Methoden in der modernen Phraseologie und Lexikographie = Corpora, Web and Databases: computer-based methods in modern phraseology and lexicography. Baltmannsweiler, Schneider Verlag Hohengehren: 197214.

\section{Wörterbücher}

Balhar, Susanne/Cyffka, Andreas (2004): PONS Großwörterbuch Deutsch als Fremdsprache. Das neue Wörterbuch für Alltag, Unterricht, Studium und Beruf. Barcelona, etc.: Klett Sprachen.

Götz, Dieter/Haensch, Günther/Wellmann, Hans (2007): Langenscheidt Großwörterbuch Deutsch als Fremdsprache. Das einsprachige Wörterbuch für alle die Deutsch lernen. Berlin, etc.: Langenscheidt.

Jones, Randall L./Tschirner, Erwin (eds.) (2006): A frequency dictionary of German. Core vocabulary for learners. London: Routledge. (=Routledge frequency dictionaries).

Kempcke, Günter (2000): Wörterbuch Deutsch als Fremdsprache. Berlin [u. a.]: de Gruyter. 
Tschirner, Erwin (2008b): Grund- und Aufbauwortschatz Deutsch als Fremdsprache nach Themen. Berlin: Cornelsen. (=Lextra).

Wahrig-Burfeind, Renate (2008): Großwörterbuch Deutsch als Fremdsprache. Gütersloh:

Wissen-Media-Verlag.

\section{Anhang 1}

\begin{tabular}{|c|c|c|c|c|c|}
\hline Sachgruppe & Rank & Basis & Kookkurrenz & log-Likelihood & $\begin{array}{l}\text { Frequenz des } \\
\text { Bigramms }\end{array}$ \\
\hline \multirow{21}{*}{$\begin{array}{l}\text { 1. Personalien, } \\
\text { Informationen zur Person }\end{array}$} & \multirow[t]{21}{*}{103} & \multirow[t]{21}{*}{ Frau } & sagen & $134^{\prime} 165^{\prime} 791$ & 3000 \\
\hline & & & sehen & $37^{\prime} 852^{\prime} 908$ & 1203 \\
\hline & & & kommen & $35^{\prime} 389^{\prime} 946$ & 1162 \\
\hline & & & lieben & $31^{\prime} 340 ' 857$ & 493 \\
\hline & & & sitzen & $28^{\prime} 414^{\prime} 541$ & 586 \\
\hline & & & gehen & $26^{\prime} 597^{\prime} 412$ & 881 \\
\hline & & & fragen & $25^{\prime} 433^{\prime} 796$ & 586 \\
\hline & & & müssen & $25^{\prime} 003^{\prime} 271$ & 1069 \\
\hline & & & stehen & $22^{\prime} 724^{\prime} 778$ & 752 \\
\hline & & & geben & $22 ' 320^{\prime} 083$ & 870 \\
\hline & & & sollen & $\underline{21^{\prime} 944^{\prime} 878}$ & 876 \\
\hline & & & lassen & $20^{\prime} 773$ '276 & 780 \\
\hline & & & wissen & $\underline{19^{\prime} 928^{\prime} 604}$ & 653 \\
\hline & & & sprechen & $17^{\prime} 557^{\prime} 015$ & 504 \\
\hline & & & rufen & $\underline{17^{\prime} 267^{\prime} 014}$ & 377 \\
\hline & & & erzählen & $\overline{15^{\prime} 953^{\prime} 965}$ & 338 \\
\hline & & & nehmen & $15^{\prime} 901^{\prime} 682$ & 545 \\
\hline & & & denken & $14^{\prime} 964^{\prime} 795$ & 440 \\
\hline & & & kennen & $11^{\prime} 388^{\prime} 860$ & 306 \\
\hline & & & dürfen & $\underline{11 ' 246 ' 830}$ & 393 \\
\hline & & & bitten & 11'079'199 & 256 \\
\hline \multirow[t]{20}{*}{ 2. Wohnen } & \multirow[t]{20}{*}{159} & \multirow[t]{20}{*}{ Haus } & kommen & 119'387'412 & 2307 \\
\hline & & & gehen & $105^{\prime} 769^{\prime} 375$ & 1968 \\
\hline & & & verlassen & 44'602'192 & 612 \\
\hline & & & stehen & $36^{\prime} 243^{\prime} 286$ & 882 \\
\hline & & & wohnen & $\underline{34^{\prime} 643^{\prime} 120}$ & 429 \\
\hline & & & bringen & $\underline{33} 821^{\prime} 387$ & 715 \\
\hline & & & bleiben & $29^{\prime} 933^{\prime} 281$ & 700 \\
\hline & & & fahren & $\underline{29^{\prime} 816^{\prime} 492}$ & 541 \\
\hline & & & bauen & $\underline{28} 145^{\prime} 964$ & 380 \\
\hline & & & müssen & $26^{\prime} 629^{\prime} 224$ & 935 \\
\hline & & & schicken & $21^{\prime} 469^{\prime} 468$ & 310 \\
\hline & & & lassen & $18^{\prime} 488^{\prime} 768$ & 617 \\
\hline & & & sehen & 17'864'077 & 645 \\
\hline & & & sitzen & $\underline{15^{\prime} 179^{\prime} 840}$ & 333 \\
\hline & & & liegen & $14^{\prime} 198^{\prime} 020$ & 421 \\
\hline & & & tragen & $13^{\prime} 922^{\prime} 524$ & 325 \\
\hline & & & sollen & 13'610'367 & 557 \\
\hline & & & treten & $133^{\prime} 036^{\prime} 831$ & 323 \\
\hline & & & nehmen & $12^{\prime} 814^{\prime} 747$ & 407 \\
\hline & & & laufen & $\underline{12} 721^{\prime} 895$ & 268 \\
\hline \multirow[t]{7}{*}{ 3. Umwelt } & \multirow[t]{7}{*}{186} & \multirow[t]{7}{*}{ Stadt } & liegen & $19^{\prime} 058^{\prime} 726$ & 472 \\
\hline & & & fahren & $16^{\prime} 097^{\prime} 847$ & 324 \\
\hline & & & kommen & 13'831'984 & 507 \\
\hline & & & gehen & $13 ' 305^{\prime} 709$ & 447 \\
\hline & & & verlassen & 13'100'391 & 227 \\
\hline & & & geben & $10^{\prime} 235^{\prime} 037$ & 420 \\
\hline & & & müssen & $8^{\prime} 249^{\prime} 442$ & 432 \\
\hline
\end{tabular}




\begin{tabular}{|c|c|c|c|c|c|}
\hline & & & sollen & $7^{\prime} 628^{\prime} 871$ & 362 \\
\hline & & & sehen & 7'578'911 & 355 \\
\hline & & & wohnen & 7'494'920 & 124 \\
\hline & & & zerstören & 7'164'856 & 112 \\
\hline & & & besetzen & 6'672'069 & 102 \\
\hline & & & ziehen & 6'136'037 & 159 \\
\hline & & & nehmen & $5^{\prime} 680^{\prime} 988$ & 226 \\
\hline & & & bauen & 5'213'734 & 100 \\
\hline & & & entstehen & 4'507'893 & 120 \\
\hline & & & bleiben & 4'376'837 & 190 \\
\hline & & & führen & 4'217'431 & 160 \\
\hline & & & bringen & 4'178'912 & 169 \\
\hline & & & stehen & $4^{\prime} 122^{\prime} 672$ & 212 \\
\hline 4. Reisen und Verkehr & 104 & Mensch & geben & & \\
\hline & & & & $59 ' 817^{\prime} 734$ & 1692 \\
\hline & & & müssen & $54^{\prime} 070 ' 781$ & 1791 \\
\hline & & & leben & 43'381'348 & 786 \\
\hline & & & sehen & $\underline{38^{\prime} 018^{\prime} 347}$ & 1271 \\
\hline & & & sollen & $\underline{31^{\prime} 918^{\prime} 789}$ & 1158 \\
\hline & & & wissen & $27^{\prime} 491^{\prime} 643$ & 842 \\
\hline & & & kommen & $24^{\prime} 437^{\prime} 007$ & 982 \\
\hline & & & sagen & 24 '208'994 & 1065 \\
\hline & & & stehen & $22 ' 248 ' 857$ & 783 \\
\hline & & & töten & $19 ' 805^{\prime} 970$ & 278 \\
\hline & & & kennen & 17'353'912 & 425 \\
\hline & & & glauben & 16'761'202 & 454 \\
\hline & & & gehen & $16^{\prime} 485^{\prime} 286$ & 700 \\
\hline & & & tuen & $16^{\prime 2} 214^{\prime} 948$ & 515 \\
\hline & & & denken & 16'170'908 & 486 \\
\hline & & & lassen & $15^{\prime} 385^{\prime} 256$ & 695 \\
\hline & & & lieben & $14^{\prime} 581^{\prime} 063$ & 290 \\
\hline & & & finden & $14^{\prime} 486 ' 459$ & 469 \\
\hline & & & brauchen & $14^{\prime} 159^{\prime} 703$ & 375 \\
\hline & & & sprechen & $14^{\prime} 055^{\prime} 984$ & 460 \\
\hline 5. Verpflegung & 970 & Essen & kommen & 7'132'043 & 175 \\
\hline & & & bringen & $6^{\prime} 440^{\prime} 157$ & 123 \\
\hline & & & einladen & 6'292'047 & 65 \\
\hline & & & geben & 6'204'988 & 158 \\
\hline & & & schlafen & $\underline{5^{\prime} 067^{\prime} 440}$ & 41 \\
\hline & & & schmecken & 4'491'352 & 46 \\
\hline & & & gehen & 4'338'075 & 115 \\
\hline & & & kochen & 4'229'942 & 45 \\
\hline & & & müssen & $\underline{3^{\prime} 962^{\prime} 158}$ & 133 \\
\hline & & & sollen & 3'789'364 & 116 \\
\hline & & & bekommen & $3^{\prime \prime 728} 006$ & 62 \\
\hline & & & laden & $3^{\prime} 6144^{\prime} 370$ & 42 \\
\hline & & & holen & $3^{\prime \prime 234 ' 165 ~}$ & 47 \\
\hline & & & lassen & $3^{\prime} 110^{\prime} 902$ & 95 \\
\hline & & & servieren & $3^{\prime} 105^{\prime} 264$ & 30 \\
\hline & & & sitzen & $2^{\prime} 879 ' 252$ & 57 \\
\hline & & & sagen & $2^{\prime} 175^{\prime} 385$ & 89 \\
\hline & & & warten & $1^{\prime} 970 ' 552$ & 35 \\
\hline & & & stehen & 1'809'865 & 62 \\
\hline & & & trinken & 1'774'439 & 29 \\
\hline 6. Einkaufen & 251 & Geld & verdienen & $73^{\prime} 555^{\prime} 996$ & 679 \\
\hline & & & kosten & $46^{\prime} 093^{\prime} 457$ & 450 \\
\hline & & & geben & $27^{\prime} 018 ' 103$ & 623 \\
\hline
\end{tabular}




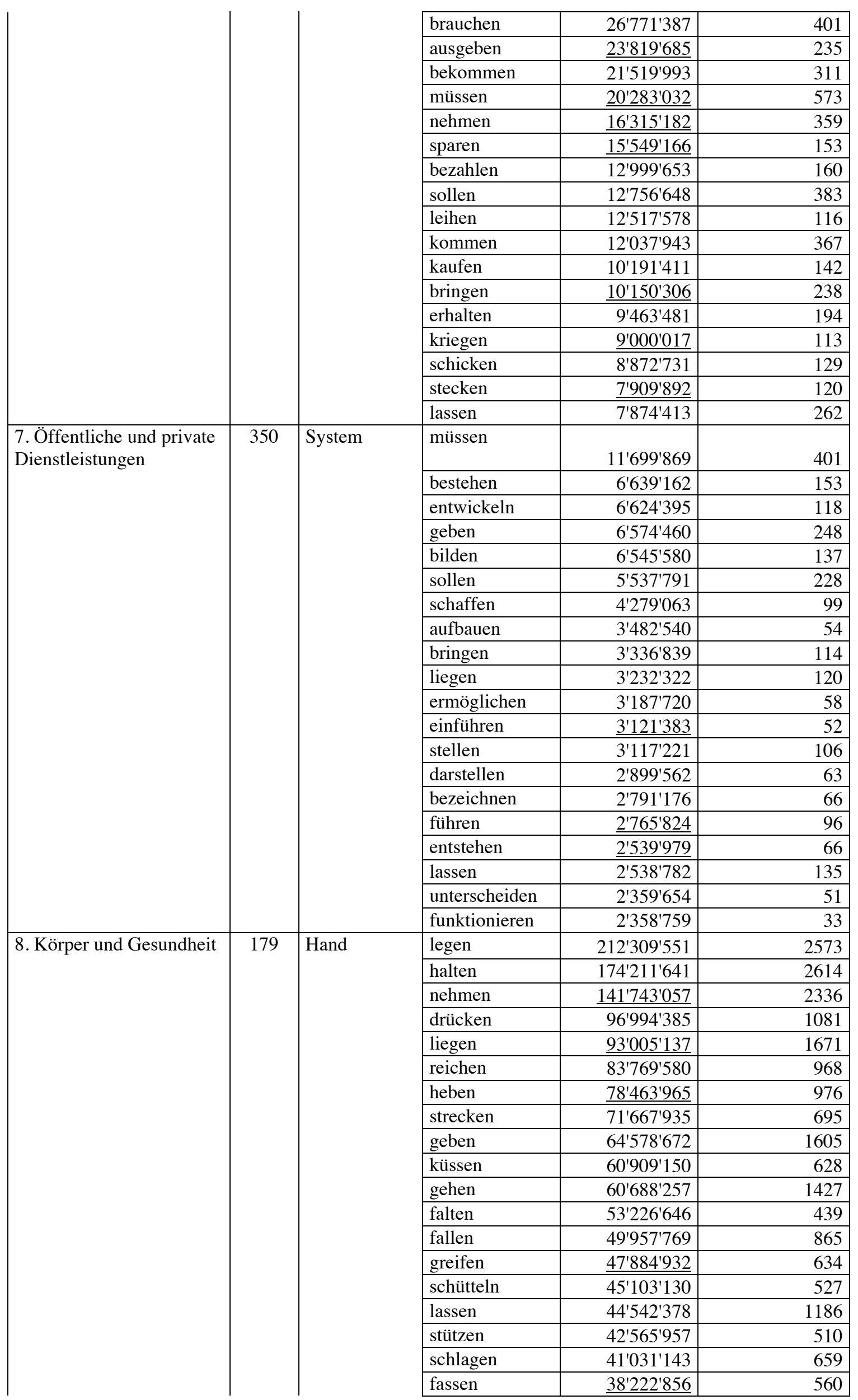




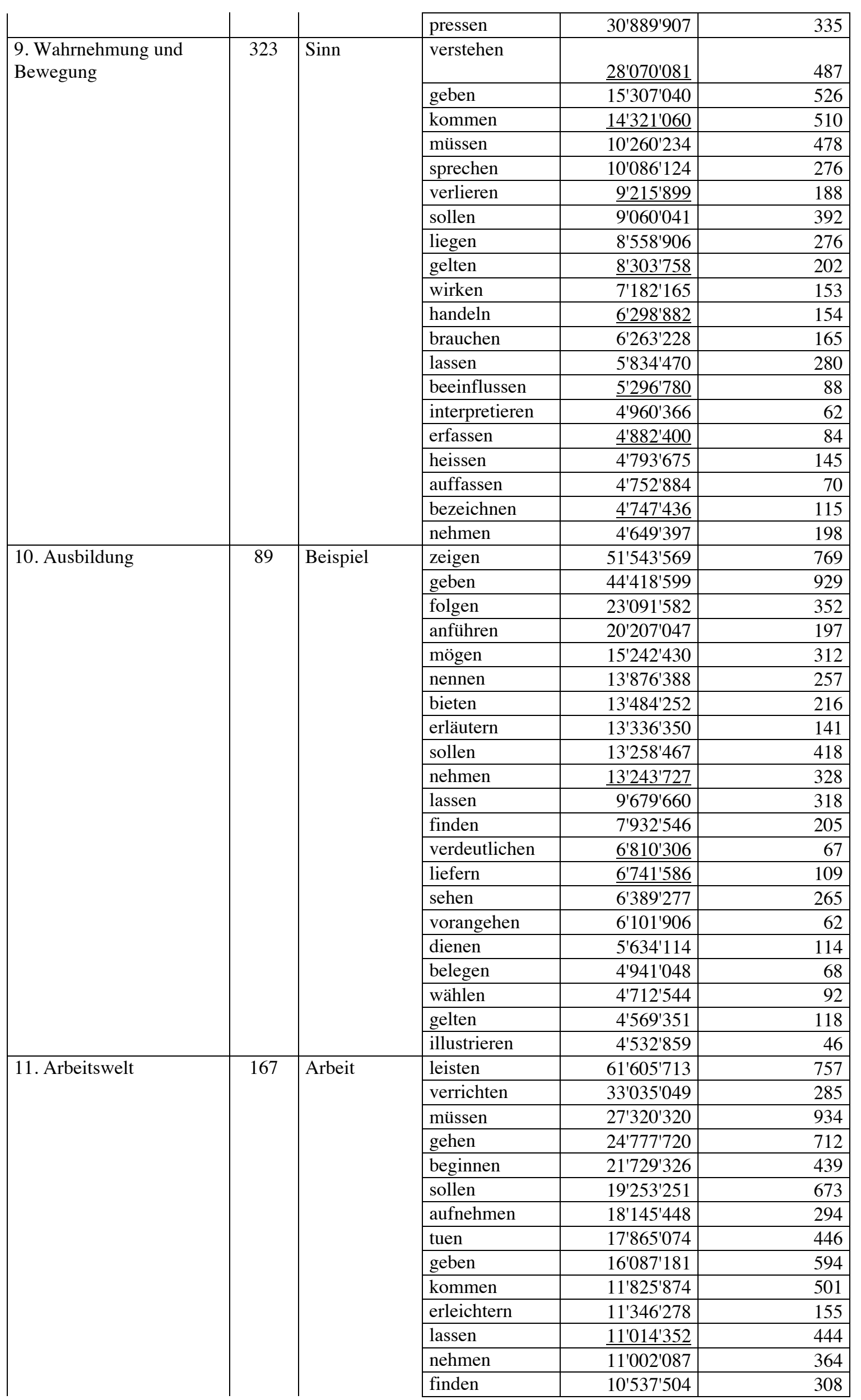




\begin{tabular}{|c|c|c|c|c|c|}
\hline \\
\hline & & & fortsetzen & $\underline{9^{\prime} 787^{\prime} 074}$ & 152 \\
\hline & & & liegen & 7'570'822 & 280 \\
\hline & & & beschäftigen & 7'554'254 & 144 \\
\hline & & & schaffen & 7'235'768 & 185 \\
\hline & & & beenden & 7'139'496 & 113 \\
\hline & & & erledigen & $66^{\prime} 519^{\prime} 020$ & 101 \\
\hline 12. Sprache & 231 & Grund & liegen & $48 ' 212^{\prime} 031$ & 945 \\
\hline & & & geben & $38^{\prime} 550^{\prime} 310$ & 1016 \\
\hline & & & müssen & 25'396'072 & 877 \\
\hline & & & nehmen & 21'472'993 & 552 \\
\hline & & & legen & $15^{\prime} 958^{\prime} 638$ & 333 \\
\hline & & & bestehen & $15^{\prime} 228^{\prime} 838$ & 347 \\
\hline & & & angeben & 14'360'188 & 194 \\
\hline & & & sollen & 13'159'713 & 526 \\
\hline & & & vorliegen & $12^{\prime} 812^{\prime} 303$ & 171 \\
\hline & & & sehen & $11^{\prime} 575 ' 248$ & 481 \\
\hline & & & erfolgen & $11^{\prime} 063^{\prime} 563$ & 214 \\
\hline & & & gehen & $11^{\prime} 038^{\prime} 621$ & 423 \\
\hline & & & dürfen & 10'941'190 & 320 \\
\hline & & & annehmen & $8^{\prime} 883^{\prime} 446$ & 187 \\
\hline & & & kommen & 8'587'781 & 410 \\
\hline & & & mögen & $\underline{8}^{\prime} 560^{\prime} 251$ & 255 \\
\hline & & & wissen & 7'430'818 & 294 \\
\hline & & & suchen & $\underline{6^{\prime} 672^{\prime} 850}$ & 176 \\
\hline & & & lassen & 6'568'599 & 325 \\
\hline & & & finden & 6'446'916 & 222 \\
\hline $\begin{array}{l}\text { 13. Freizeit und } \\
\text { Unterhaltung }\end{array}$ & 204 & Ende & gehen & $51^{\prime} 564 ' 336$ & 1071 \\
\hline & & & bereiten & $41^{\prime} 552 ' 568$ & 445 \\
\hline & & & führen & 37'664'382 & 678 \\
\hline & & & nehmen & 32'306'943 & 680 \\
\hline & & & setzen & $24^{\prime} 039^{\prime} 822$ & 461 \\
\hline & & & kommen & $22^{\prime} 727^{\prime} 053$ & 668 \\
\hline & & & müssen & $22^{\prime} 4466^{\prime} 406$ & 742 \\
\hline & & & stehen & $18^{\prime} 920^{\prime} 872$ & 512 \\
\hline & & & finden & 17'903'639 & 401 \\
\hline & & & sollen & 17'791'017 & 575 \\
\hline & & & bringen & $13^{\prime} 002^{\prime} 605$ & 334 \\
\hline & & & betragen & $12^{\prime} 724^{\prime} 678$ & 199 \\
\hline & & & erreichen & $11 ' 312 ' 240$ & 229 \\
\hline & & & denken & $\underline{10^{\prime} 027 ' 390}$ & 262 \\
\hline & & & beginnen & 9'216'034 & 221 \\
\hline & & & erfinden & 8'483'091 & 193 \\
\hline & & & bedeuten & 7'883'894 & 164 \\
\hline & & & sehen & 7'691'650 & 344 \\
\hline & & & bleiben & 7'385'524 & 250 \\
\hline & & & geben & 5'957'199 & 298 \\
\hline 14. Persönliche & 305 & Entwicklung & fördern & & \\
\hline Kontakte & & & & $20 ' 068 ' 729$ & 256 \\
\hline & & & laufen & $\underline{11 ' 804^{\prime} 685}$ & 225 \\
\hline & & & zeigen & $11 ' 346 ' 034$ & 273 \\
\hline & & & verfolgen & 9'039'174 & 140 \\
\hline & & & beitragen & $8^{\prime} 662^{\prime} 697$ & 126 \\
\hline & & & hemmen & $8 ' 523 ' 943$ & 93 \\
\hline & & & vollziehen & 8'439'918 & 124 \\
\hline & & & führen & $\underline{8^{\prime} 254^{\prime} 703}$ & 227 \\
\hline
\end{tabular}




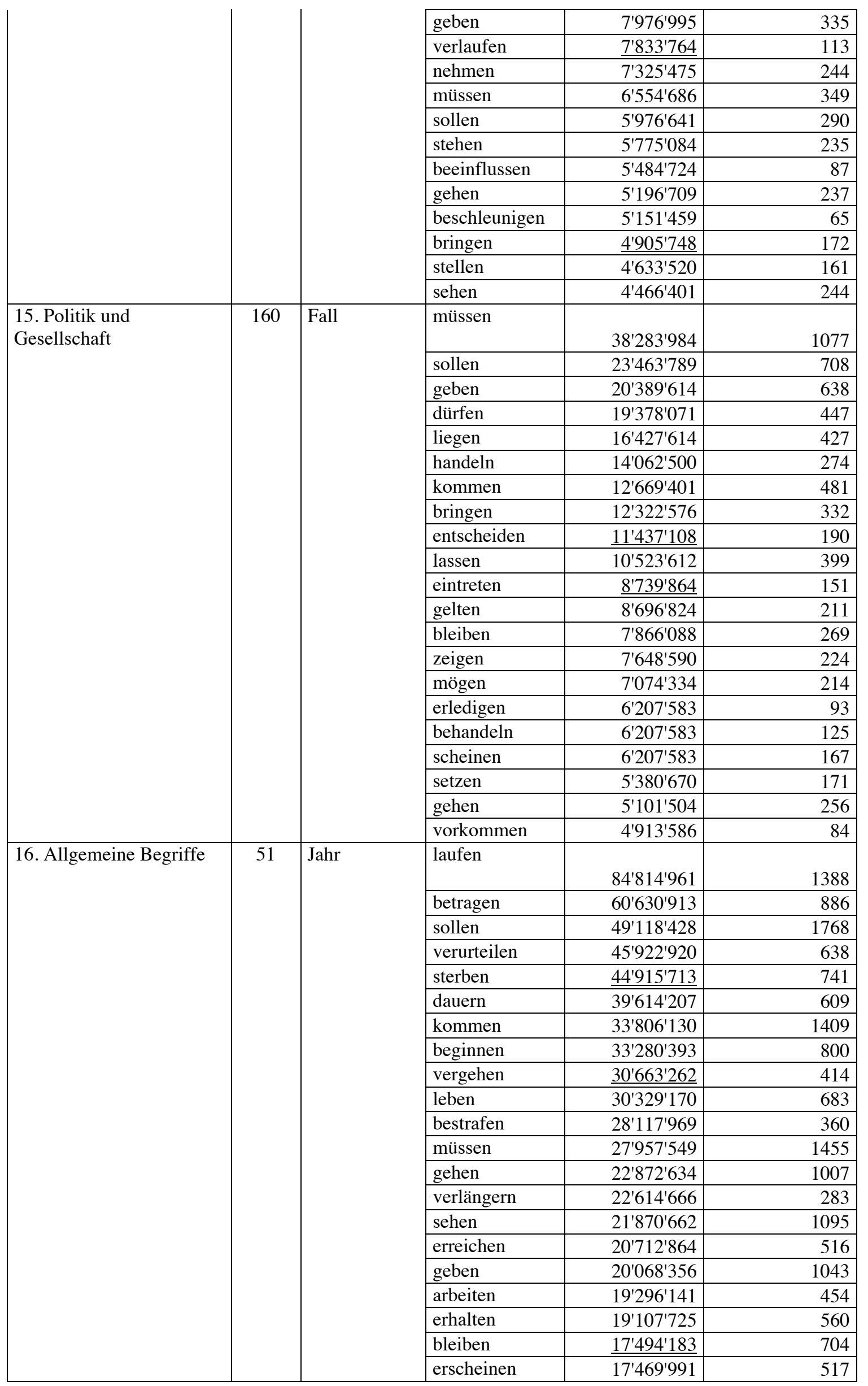


Anhang 2

\begin{tabular}{|c|c|c|c|c|}
\hline Rank & Basis & Kookkurrenz & log-Likelihood & Frequenz des Bigramms \\
\hline \multirow[t]{20}{*}{46} & \multirow[t]{20}{*}{ sagen } & Herr & 220570840 & 4354 \\
\hline & & Frau & 134165791 & 3000 \\
\hline & & Mutter & 103583018 & 1950 \\
\hline & & Wort & 85631719 & 2001 \\
\hline & & Vater & 83451514 & 1683 \\
\hline & & Wahrheit & 63520132 & 1088 \\
\hline & & Stimme & 56004976 & 1204 \\
\hline & & Fräulein & 43393301 & 705 \\
\hline & & Gott & 35065608 & 957 \\
\hline & & Doktor & 29545967 & 516 \\
\hline & & Kind & 29481714 & 1025 \\
\hline & & Hand & 28329409 & 1027 \\
\hline & & Weile & 26333728 & 453 \\
\hline & & Mensch & 24208994 & 1065 \\
\hline & & Mama & 22827866 & 358 \\
\hline & & Papa & 22806660 & 345 \\
\hline & & Onkel & 22645410 & 403 \\
\hline & & Junge & 22132676 & 478 \\
\hline & & Tante & 22039871 & 381 \\
\hline & & Freund & 22025627 & 584 \\
\hline \multirow[t]{20}{*}{57} & \multirow[t]{20}{*}{ geben } & Antwort & 153416191 & 2036 \\
\hline & & Möglichkeit & 127951172 & 1998 \\
\hline & & Ausdruck & 153416191 & 1796 \\
\hline & & Wehrmacht & 102610625 & 1170 \\
\hline & & Anlass & 97643662 & 1228 \\
\hline & & Auskunft & 95389697 & 1008 \\
\hline & & Mühe & 79716714 & 988 \\
\hline & & Oberkommando & 79086260 & 789 \\
\hline & & Gelegenheit & 76012012 & 1119 \\
\hline & & Hand & 64578672 & 1605 \\
\hline & & Mensch & 59817734 & 1692 \\
\hline & & Hauptquartier & 47651440 & 512 \\
\hline & & Erklärung & 45337837 & 847 \\
\hline & & Befehl & 44987944 & 698 \\
\hline & & Beispiel & 44418599 & 929 \\
\hline & & Leute & 43939663 & 963 \\
\hline & & Zeit & 40700010 & 1419 \\
\hline & & Grund & 38550310 & 1016 \\
\hline & & Aufschluss & 38427673 & 399 \\
\hline & & Regierung & 37124590 & 1089 \\
\hline \multirow[t]{14}{*}{81} & \multirow[t]{14}{*}{ sehen } & Auge & 178515391 & 3042 \\
\hline & & Abschnitt & 110688955 & 1414 \\
\hline & & Gesicht & 104936143 & 1806 \\
\hline & & Seite/S. & 66983037 & 1507 \\
\hline & & Fenster & 60879512 & 1006 \\
\hline & & Mann & 46027158 & 1358 \\
\hline & & Wortlaut & 44085264 & 548 \\
\hline & & Mensch & 38018347 & 1271 \\
\hline & & Frau & 37852908 & 1203 \\
\hline & & Bild & 34198662 & 840 \\
\hline & & Blick & 28878391 & 652 \\
\hline & & Mal & 27797781 & 574 \\
\hline & & Herr & 27479351 & 1017 \\
\hline & & Übersicht & 24956365 & 334 \\
\hline
\end{tabular}




\begin{tabular}{|c|c|c|c|c|}
\hline & & Hand & 24062534 & 852 \\
\hline & & Abkommen & 23768650 & 484 \\
\hline & & Jahr & 21870662 & 1095 \\
\hline & & Kind & 21355398 & 772 \\
\hline & & Mutter & 21140647 & 609 \\
\hline & & Leute & 21122942 & 587 \\
\hline \multirow[t]{20}{*}{82} & \multirow[t]{20}{*}{ lassen } & Stich & 71145850 & 715 \\
\hline & & Ruhe & 49941216 & 753 \\
\hline & & Zeit & 45984380 & 1439 \\
\hline & & Hand & 44542378 & 1186 \\
\hline & & Zweifel & 31950046 & 516 \\
\hline & & Auge & 27242197 & 776 \\
\hline & & Wort & 26701865 & 810 \\
\hline & & Kind & 23541633 & 768 \\
\hline & & Herr & 21737927 & 826 \\
\hline & & Frau & 20773276 & 780 \\
\hline & & Wasser & 19643469 & 495 \\
\hline & & Kopf & 18814764 & 538 \\
\hline & & Tag & 18756415 & 736 \\
\hline & & Haus & 18488768 & 617 \\
\hline & & Mann & 18387510 & 723 \\
\hline & & Arm & 17441212 & 422 \\
\hline & & Blick & 16746040 & 426 \\
\hline & & Leben & 16372444 & 629 \\
\hline & & Mensch & 15385256 & 695 \\
\hline & & Minute & 15277977 & 335 \\
\hline \multirow[t]{20}{*}{112} & \multirow[t]{20}{*}{ bleiben } & Zeit & 38722732 & 1059 \\
\hline & & Haus & 29933281 & 700 \\
\hline & & Tag & 24181233 & 698 \\
\hline & & Leben & 22336660 & 621 \\
\hline & & Frage & 20826824 & 601 \\
\hline & & Jahr & 17494183 & 74 \\
\hline & & Weile & 16874061 & 258 \\
\hline & & Kraft & 16026349 & 398 \\
\hline & & Tür & 13734359 & 304 \\
\hline & & Erinnerung & 13390293 & 242 \\
\hline & & Nacht & 12358243 & 288 \\
\hline & & Gedächtnis & 12303071 & 181 \\
\hline & & Mensch & 11868616 & 473 \\
\hline & & Kind & 11343202 & 396 \\
\hline & & Erfolg & 11288390 & 255 \\
\hline & & Land & 9672659 & 382 \\
\hline & & Antwort & 9656190 & 218 \\
\hline & & Stunde & 9292119 & 264 \\
\hline & & Platz & 9047001 & 228 \\
\hline & & Rest & 8957357 & 166 \\
\hline \multirow[t]{11}{*}{118} & \multirow[t]{11}{*}{ liegen } & Bett & 101597676 & 1229 \\
\hline & & Hand & 93005137 & 1671 \\
\hline & & Interesse & 53970610 & 903 \\
\hline & & Grund & 48212031 & 945 \\
\hline & & Boden & 42970723 & 703 \\
\hline & & Ding & 33147336 & 621 \\
\hline & & Herz & 27634282 & 505 \\
\hline & & Tisch & 27166692 & 476 \\
\hline & & Schnee & 23805476 & 308 \\
\hline & & Verhältnis & 22116333 & 460 \\
\hline & & Schwerpunkt & 21691941 & 233 \\
\hline
\end{tabular}




\begin{tabular}{|c|c|c|c|c|}
\hline & & \multirow{2}{*}{$\begin{array}{l}\text { Auge } \\
\text { Stadt }\end{array}$} & \multirow{2}{*}{$\begin{array}{l}19780115 \\
10058726\end{array}$} & 515 \\
\hline & & & & 472 \\
\hline & & Seite & 18324346 & 491 \\
\hline & & Tag & 18145867 & 574 \\
\hline & & Rücken & 17339091 & 279 \\
\hline & & Fall & 16427614 & 427 \\
\hline & & Sache & 16383866 & 379 \\
\hline & & Natur & 16346592 & 344 \\
\hline & & Straße & 16258680 & 345 \\
\hline \multirow[t]{20}{*}{123} & \multirow[t]{20}{*}{ heißen } & Name & 2035987 & 40 \\
\hline & & Gott & 1463462 & 33 \\
\hline & & Vorname & 1194234 & 12 \\
\hline & & Vorsitzende & 1052403 & 20 \\
\hline & & Herr & 966955 & 33 \\
\hline & & Menge & 959753 & 18 \\
\hline & & Wort & 932702 & 28 \\
\hline & & Mensch & 882584 & 32 \\
\hline & & Mann & 872682 & 30 \\
\hline & & Wahrheit & 855157 & 17 \\
\hline & & Leute & 842901 & 21 \\
\hline & & Kind & 700008 & 24 \\
\hline & & Sohn & 691752 & 16 \\
\hline & & Ding/Dinger & 643258 & 17 \\
\hline & & Dame & 634097 & 14 \\
\hline & & Vater & 625088 & 18 \\
\hline & & S./Seite & 619011 & 22 \\
\hline & & Sprache & 605211 & 15 \\
\hline & & Prophet & 582440 & 8 \\
\hline & & Philosoph & 581825 & 9 \\
\hline \multirow[t]{20}{*}{124} & \multirow[t]{20}{*}{ denken } & Mensch & 16170908 & 486 \\
\hline & & Frau & 14964795 & 440 \\
\hline & & Gott & 14337119 & 336 \\
\hline & & Augenblick & 12524241 & 255 \\
\hline & & Mann & 11160087 & 364 \\
\hline & & Zeit & 10461278 & 398 \\
\hline & & Herr & 10226084 & 356 \\
\hline & & Ende & 10027390 & 262 \\
\hline & & Vater & 9380975 & 244 \\
\hline & & Wort & 9071987 & 286 \\
\hline & & Leute & 8408050 & 217 \\
\hline & & Tag & 8197442 & 303 \\
\hline & & Mutter & 8036255 & 218 \\
\hline & & Kind & 7453533 & 260 \\
\hline & & Gedanke & 7035423 & 180 \\
\hline & & Ding & 6421656 & 176 \\
\hline & & Junge & 5237881 & 116 \\
\hline & & Mädchen & 4848050 & 130 \\
\hline & & Leben & 4685704 & 202 \\
\hline & & Fräulein & 4262822 & 90 \\
\hline \multirow[t]{8}{*}{140} & \multirow[t]{8}{*}{ tun } & Schritt & 70995879 & 959 \\
\hline & & Pflicht & 45551025 & 589 \\
\hline & & Gefallen & 42794463 & 362 \\
\hline & & Dienst & 28546565 & 427 \\
\hline & & Abbruch & 25441919 & 244 \\
\hline & & Arbeit & 17865074 & 446 \\
\hline & & Unrecht & 16878691 & 211 \\
\hline & & Schuldigkeit & 16518082 & 130 \\
\hline
\end{tabular}




\begin{tabular}{|c|c|c|c|c|}
\hline & & \multirow{2}{*}{$\begin{array}{l}\text { Mensch } \\
\text { Genüge }\end{array}$} & 16214948 & 515 \\
\hline & & & 15970925 & 150 \\
\hline & & Gut(es) & 14418995 & 233 \\
\hline & & Sache & 14104873 & 318 \\
\hline & & Herr & 13292584 & 444 \\
\hline & & Mann & 10783943 & 378 \\
\hline & & Ding & 10712009 & 261 \\
\hline & & Einhalt & 9553929 & 92 \\
\hline & & Frau & 8996276 & 340 \\
\hline & & Gott & 8271319 & 245 \\
\hline & & Kind & 7847465 & 285 \\
\hline & & Leute & 7612448 & 214 \\
\hline \multirow[t]{19}{*}{143} & \multirow[t]{19}{*}{ glauben } & Gott & 19471842 & 386 \\
\hline & & Herr & 16914363 & 447 \\
\hline & & Mensch & 16761202 & 454 \\
\hline & & Leute & 11755490 & 252 \\
\hline & & Irrtum & 7340773 & 102 \\
\hline & & Frau & 6646188 & 240 \\
\hline & & Grund & 6215082 & 190 \\
\hline & & Vorsitzende & 6083627 & 129 \\
\hline & & Zeit & 5678364 & 250 \\
\hline & & Augenblick & 4991360 & 124 \\
\hline & & Wunder & 4822929 & 81 \\
\hline & & Regierung & 4680916 & 177 \\
\hline & & Volk & 4072032 & 146 \\
\hline & & Welt & 3712835 & 151 \\
\hline & & Wahrheit & 3696182 & 90 \\
\hline & & Leben & 3670696 & 157 \\
\hline & & Recht & 3470337 & 124 \\
\hline & & Sache & 3384304 & 107 \\
\hline & & Sieg & 3326099 & 70 \\
\hline
\end{tabular}

